\begin{tabular}{|c|c|}
\hline & $\begin{array}{l}\text { International Journal of Trend in Scientific } \\
\text { Research and Development (IJTSRD) }\end{array}$ \\
\hline 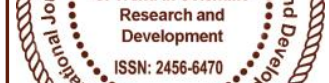 & International Open Access Journal \\
\hline & ISSN No: 2456 - 6470 | www.ijtsrd.com | Volume - 2 | Issue - 2 \\
\hline
\end{tabular}

\title{
An Efficient Brain Tumor Detection Algorithm based on Segmentation for MRI System
}

\author{
Merlin Asha. M¹, G. Naveen Balaji ${ }^{2}$, S. Mythili², A. Karthikeyan², N. Thillaiarasu ${ }^{3}$ \\ ${ }^{1}$ PG Student, Department of ECE, SNS College of Technology, Coimbatore, India \\ ${ }^{2}$ Assistant Professor, Department of ECE, SNS College of Technology, Coimbatore, India \\ ${ }^{3}$ Assistant Professor, Department of CSE, SNS College of Engineering, Coimbatore, India
}

\section{ABSTRACT}

A collection, or mass, of abnormal cells in the brain is called as Brain Tumor. The skull, which encloses your brain, is very rigid. Growth inside such a restricted space can cause problems. Brain tumors can be malignant or benign. Segmentation in magnetic resonance imaging (MRI) was an emergent research area in the field of medical imaging system. In this an efficient algorithm is proposed for tumor detection based on segmentation and morphological operators. Quality of scanned image is enhanced and then morphological operators are applied to detect the tumor in the scanned image.

Keywords: Brain Tumor, MRI, Morphological Operators, Segmentation, Detection

\section{INTRODUCTION}

3D segmentation of images of medical imaging plays a vital role in stages which occur before implementing object recognition. 3D image segmentation helps in automated diagnosis of brain diseases and helps in qualitative and quantitative analysis of images such as measuring accurate size and volume of detected portion.

Accurate measurements are quite difficult in brain diagnosis because of diverse shapes, sizes and appearances of tumors. Tumors can grow abruptly causing defects in neighboring tissues also, which gives an overall abnormal structure for healthy tissues as well. Here, a technique is developed for 3D segmentation of a brain tumor by using segmentation in conjunction with morphological operations.

\section{A. Tumor:}

The word tumor is also known as neoplasm which is formed by an abnormal growth of cells. It is something totally different from cancer.

\section{1) Types of Tumor:}

There are three common types of tumor [1]:

a) Benign Tumor: This is a tumor is the one that does not expand in an abrupt way. It doesn't affect its neighboring healthy tissues and also does not expand to non-adjacent tissues. Moles are the common example of this tumors.

b) Pre-Malignant Tumor: It is considered as a disease, a precancerous stage, if not properly treated it may lead to cancer.

c) Malignant Tumor Malignancy (mal- = "bad" and ignis = "fire"): It is the type of tumor, that grows worse with the passage of time and ultimately results in the death. Typically the term, Malignant tumor is used for the description of cancer.

\section{B. Magnetic Resonance Imaging (MRI):}

Strong magnetic field is used in MRI to align the nuclear magnetization then radio frequencies changes the alignment of the magnetization which can be 
detected by the scanner. Whereas CT uses ionizing radiation. And the signal can be further processed to create the extra information of the body.

This paper is divided into six parts, where in the second part analysis and findings are discussed. In the third portion related work is explained, many researchers are currently working in this field. Many related research papers are properly explained in this region. Forth portion is proposed methodology, all the phases of our proposed system is explained in details.

\section{ANALYSIS AND FINDINGS}

Brain tumor is one of the main cause for increasing mortality among children and adults. The researchers of the most developed countries has concluded that number of people suffering and dying from brain tumors has been increased to 300 per year during past few decades. Bar Graph is given below; showing number of diagnosed and died of tumor.
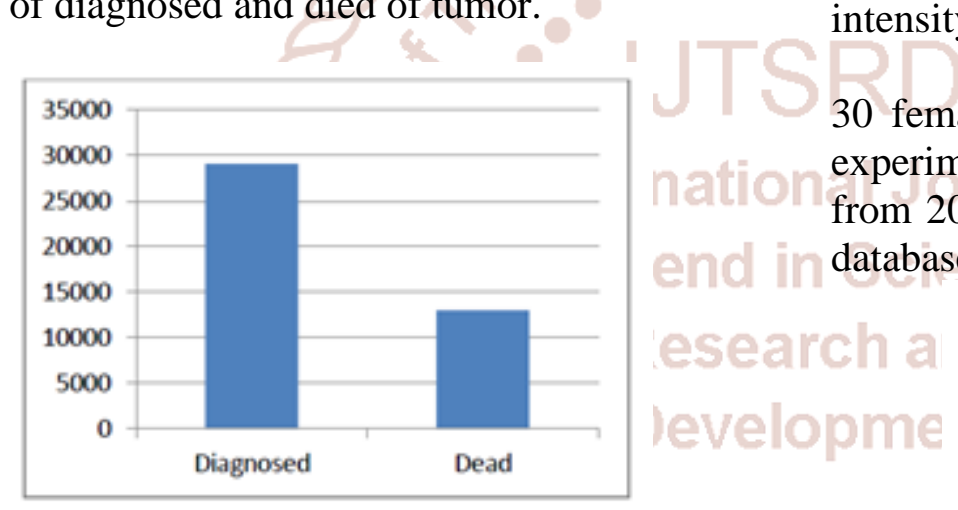

Figure 1: Analysis of Tumor Diagnosis [9]

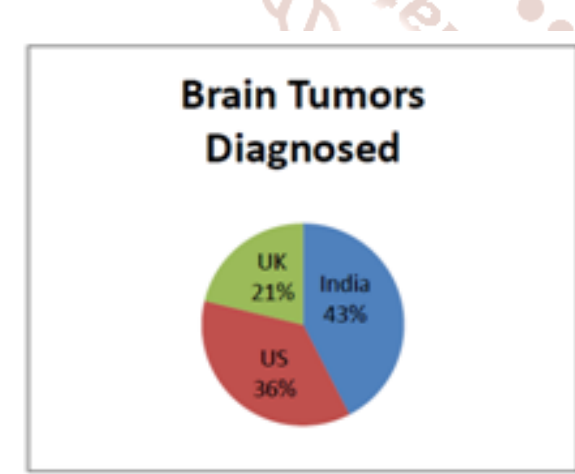

Figure 2: Rate of Tumor Diagnosis Per Year[9]

\section{RELATED WORK}

The process where an image is divided into the different regions on some similarity bases is called Segmentation. Basic function is that we can easily extract the image information and different features. Medical experts do the brain tumor detection because it is very time consuming process. [3] Many of these techniques are not properly defined rather they are ad hoc techniques.

Some of the most common methods are:

1) Amplitude thresholding;

2) Texture segmentation;

3) Template matching;

4) Region-growing segmentation

\section{PROPOSED METHODOLOGY}

\section{A. Image Acquisition:}

Images for this are obtained using MRI scan and these scanned images are displayed in a two dimensional matrices having pixels as its elements. These matrices are dependent on the matrix size and its field of view. In MATLAB, the images are stored and displayed as a gray scale image of size $256 * 256$. The entries of a gray scale image are ranging from 0 to 255 , where 0 indicates total black color and 255 indicates pure white color. Entries between this range vary in intensity from black to white.

30 female and 30 male patients were examined for experimental purpose, all patients have ages ranging from 20 to 60 years. Their MRI scans were stored in database of images in JPEG image formats.

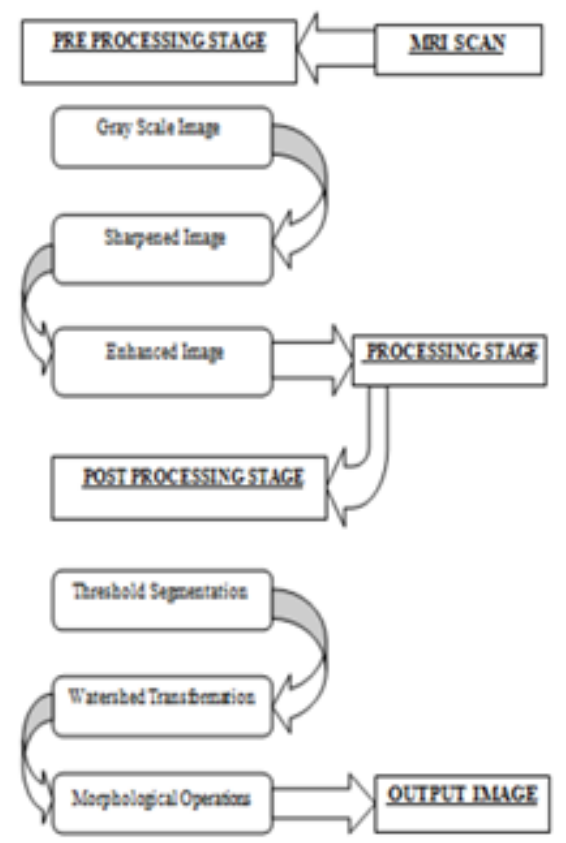

Figure3: Stages of Tumor Detection

\section{B. Preprocessing:}

The image in this phase is enhanced in the way that finer details are improved and noise is removed from the image. Most commonly used enhancement and 
noise reduction techniques are implemented that can give best possible results.

The more prominent edges and a sharpened image is obtained as the result of the enhancement, the noise will be reduced thus reducing the blurring effect from the image. In addition to enhancement, image segmentation will also be applied. This improved and enhanced image will help in detecting edges and improving the quality of the overall image. Finding the exact location of tumor is done by Edge detection. Following steps will be followed in the preprocessing stage:

The acquired MRI scanned image, stored in database is converted to gray scale image of size $255^{*} 255$; 2) image is processed to remove any noise represent. Visual quality of noisy image will not be satisfactory; 3 ) the noiseless, high quality image is then operated by a high pass filter for sharpening and edge detection. ; 4) The obtained sharpened image is then added to original image for further enhancement.

a) Noise Removal: Many filters are used to remove the noise from the images. Linear filters can also serve the purpose like Gaussian, averaging filters. For example average filters are used to remove salt and pepper noise from the image. Because in this filter pixel's value is replaced with its neighborhood values. b) Image Sharpening: Sharpening of the image can be achieved by using different high pass filters. By using different low pass filters, noise is been removed. We need to sharpens the image as we need the sharp edges because this will help us to detect the boundary of the tumor.

\section{Processing:}

1) Segmentation: Image segmentation is based on the division of the image into regions. Division is done on the basis of similar attributes. Similarities are separated out into groups. Ultimate purpose of segmentation is the extraction of important features from the image, from which information can easily be perceived. Brain tumor segmentation from MRI images is an interesting but challenging task in the field of medical imaging.

\section{Post-Processing:}

In processing segmentation is done using following methods.

1) Threshold Segmentation: It is the simplest method and the input gray scale image is converted into a binary format. The method is based on a threshold value which will convert gray scale image into a binary image format. The main logic is the selection of a threshold value. Few methods used under this segmentation use maximum entropy method and kmeans clustering method for segmentation.[10]

2) Watershed Segmentation: To group pixels of an image on the basis of their intensities is the best methods. Pixels with similar intensities are grouped together. This is the predominant segmentation technique for dividing an image to separate a tumor from the image. Watershed is a mathematical morphological operating tool.

For using watershed segmentation different methods are used. By the segmentation technique output is checked only because it usually suffers from over segmentation and under segmentation. Two basic principle techniques are given below: 1) the computed local minima of the image gradient are chosen as a marker. In this method an over segmentation occurs.

2) Watershed utilizes the specifically defined marker positions transformation using markers. These positions are defined explicitly by a user and also by using morphological tools automatically they can be determined.

3) Morphological Operators: After converting the image in the binary format, some morphological operations are applied on the converted binary image. The predominant work of the morphological operators is to separate the tumor part of the image. Now only the tumor portion of the image is visible, shown as white color. This portion has the highest intensity than other regions of the image.

Commands used in morphing are given below: 1) Strel: Used for creating morphological structuring element; 2) Imerode():Used to erode (Shrink) an image.[12] ;3)Imdilate():Used for dilating (filling, expanding) an image.[12]

\section{EXPERIMENTAL OUTCOMES}

First of all input image is shown here, figure 4 shows input images which has brain tumor. Threshold segmentation is applied on these images which contains brain tumor. The results are shown in the figure 5 . 

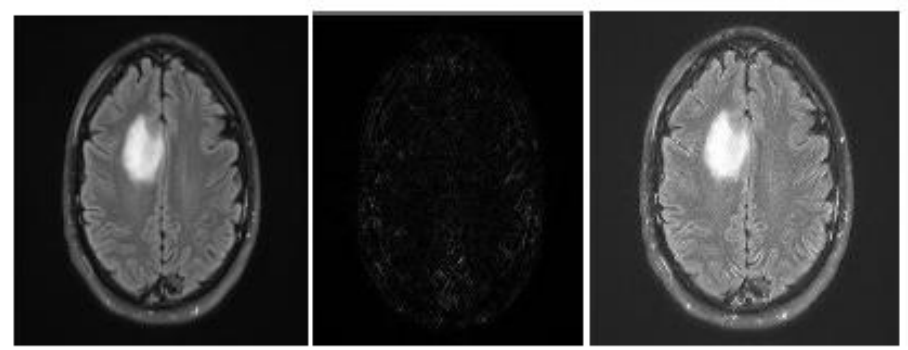

Figure 4: Input Brain Image

In the following figure white spot is shown, which is the result of threshold segmentation applied on the images. This is basically the area with the intensity values higher than the defined threshold. High intensity areas mostly comprises of tumors. So through threshold segmentation we can specify the location of tumor.

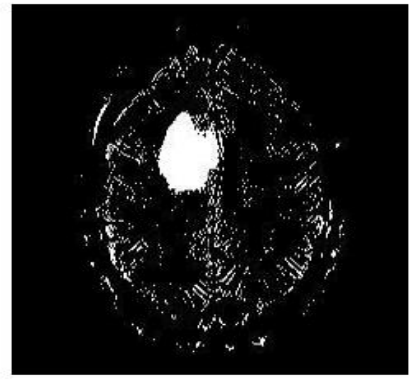

Figure 5: Threshold Segmentation

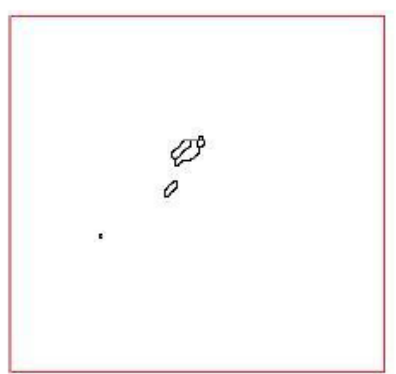

Figure 6: Watershed Segmentation

Watershed segmentation ia a technique which is applied on the resulted image obtained after threshold segmentation. Only the portion which contains tumor is highlighted here. The portions with the high intensity values are detected through threshold segmentation. These portions are marked through watershed segmentation methods. Morphological operators are applied after the watershed segmentation. This technique is very efficient and the results are little prominent.
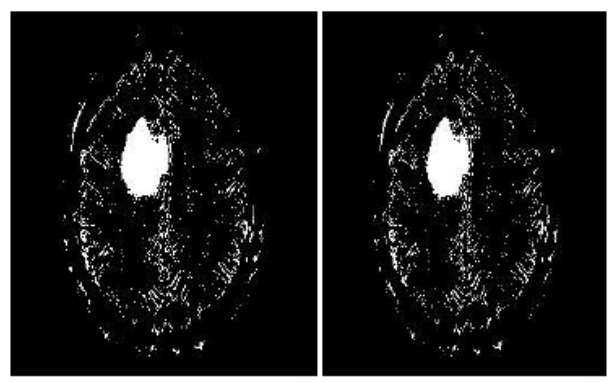

Figure 7: Morphological Operators

Figure 8: contains the resulted image obtained after applying functions like erosion.

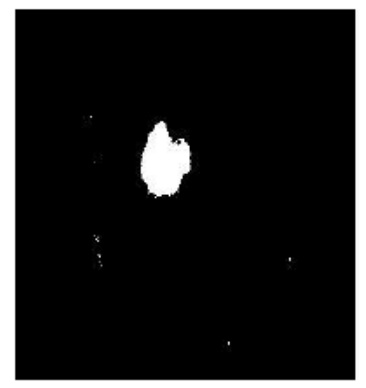

Figure 8: Imerode With strel('disk',1)

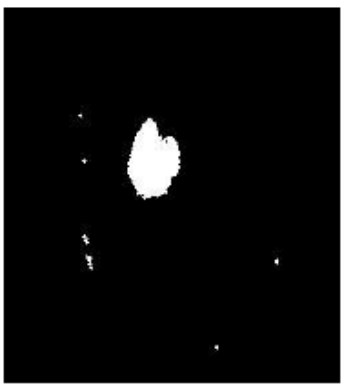

Figure 9: Imdilate With strel('disk',1)

Figure 9 contains the resulted image of dilation function which is applied after the erosion. 


\section{A. Output Image:}

Tumor is displayed as white portion in the image.
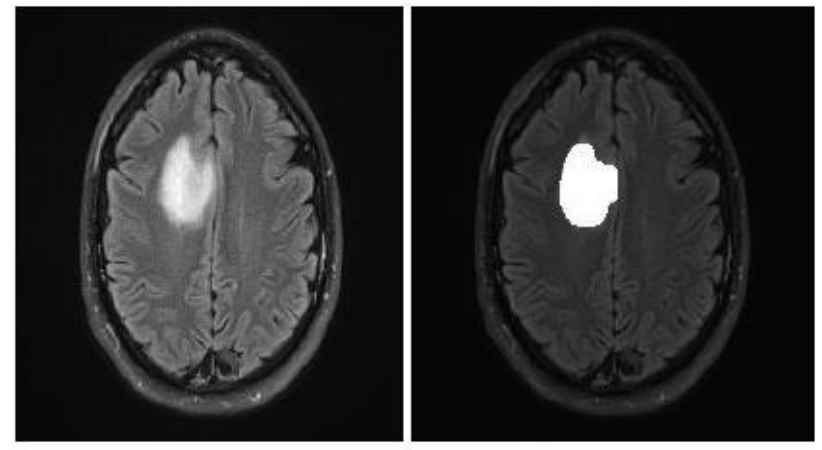

Figure 10: Tumor Detected As White Portion

\section{CONCLUSION AND FUTURE WORK}

Using Medical Imaging techniques the research was conducted to detect the brain tumor. The main technique used was segmentation, which is done using a method based on threshold segmentation, watershed segmentation and morphological operators. Using MRI scanned images of human brains the proposed segmentation technique was experimented: thus locating tumor in the images. Samples of human brains were taken, scanned using MRI process and then were processed through segmentation methods thus giving efficient end results.

This technique gives efficient results as compared to previous researches. Experiments are applied on various images and results were extraordinary. Our proposed research is easy to execute and thus can be managed easily.

Our future work is to extend our proposed method for color based segmentation of 3D images. For this purpose we need a classification method to organize three dimensional objects into separate feature classes, whose characteristics can help in diagnosis of brain diseases.

\section{REFERENCES}

1. Oelze, M.L,Zachary, J.F. , O'Brien, W.D., Jr., -Differentiation of tumor types in vivo by scatterer property estimates and parametric images using ultrasound backscatter — , on page(s) :1014 - 1017 Vol.1, 5-8 Oct. 2003.

2. Devos, A, Lukas, L., -Does the combination of magnetic resonance imaging and spectroscopic imaging improve the classification of brain tumours?\|, On Page(s): 407 - 410, Engineering in
Medicine and Biology Society, 2004. IEMBS '04. 26th Annual International Conference of the IEEE, 1-5 Sept. 2004.

3. Farmer, M.E, Jain, A.K. , -A wrapper-based approach to image segmentation and classificationl, Page(s): 2060 - 2072 , Image Processing, IEEE Transactions on journals and magazines, Dec. 2005.

4. Gopal,N.N. Karnan, M. , -Diagnose brain tumor through MRI using image processing clustering algorithms such as Fuzzy $\mathrm{C}$ Means along with intelligent optimization techniques $-, \operatorname{Page}(\mathrm{s}): 1$ - 4, Computational Intelligence and Computing Research (ICCIC), 2010 IEEE International Conference, 28-29 Dec. 2010.

5. Joshi, D.M.; Rana, N.K.; Misra, V.M. i ,\| Classification of Brain Cancer using Artificial Neural Network - , Page(s): 112 - 116, Electronic Computer Technology (ICECT), 2010 International Conference, 7-10 May 2010

6. Thillaiarasu N.Chenthur Pandian S 2017, "A Novel Scheme for Safeguarding Confidentiality in Public Clouds for Service Users of Cloud Computing", Cluster Computing-The Journal of Networks, Software Tools and Applications, Springer.DOI: 1386-7857 1573-7543.ISSN: 15737543.

7. N.Thillaiarasu, Dr.S.Chenthur Pandian "Enforcing Security and Privacy over Multi- loud Framework Using Assessment Techniques", International Conference on Intelligent Systems and Control (ISCO16), DOI: 10.1109/ISCO.2016.7727001,IEEE Explore. 
8. N.Shyamambika, N.Thillaiarasu "A Survey on Acquiring Integrity of Shared Data with Effective user Termination in the Cloud"International Conference on Intelligent Systems and Control (ISCO16), DOI: 10.1109/ISCO.2016.7726893,IEEE Explore.

9. N.Shyamambika,N.Thillaiarasu,"Attaining Integrity, Secured Data Sharing and Removal of Misbehaving Client in the Public Cloud using an External Agent and Secure Encryption Technique", Advances in Natural and Applied Sciences. 10(9); Pages: 421-431.

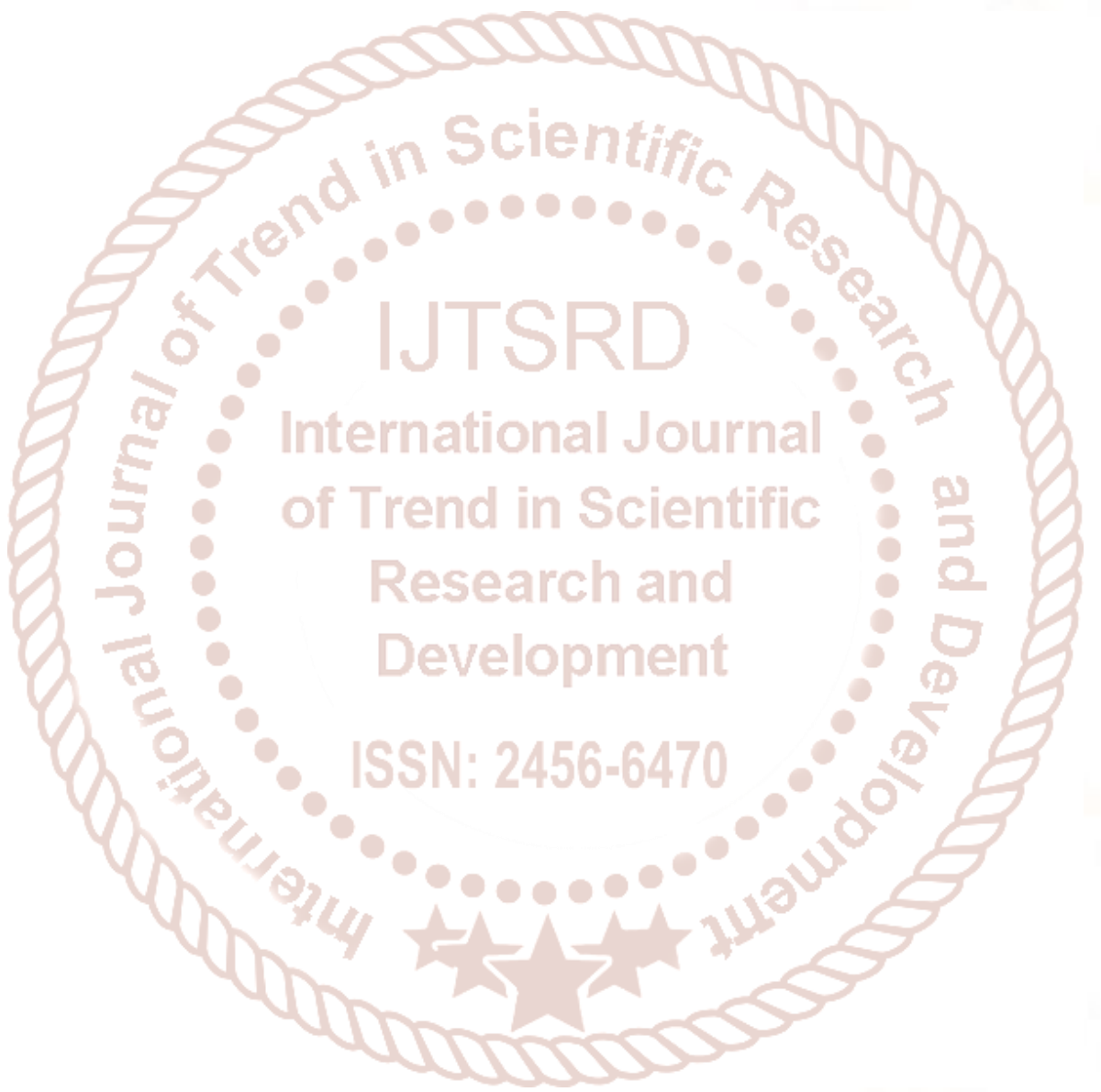

